\title{
Quelle stratégie de change dans les économies en développement ? Thierry Montalieu ${ }^{(*)}$
}

Le taux de change est un instrument central des stratégies d'ajustement de la balance des paiements. Dans ce contexte, il est pertinent de s'interroger sur le choix qui est fait en matière de régime de change par les pays en développement (PED) et sur ce qui doit motiver ce choix. L'efficacité d'un régime de change se mesure traditionnellement à travers deux critères : la solidité et l'instabilité. Pour le premier, il s'agit de déterminer la capacité d'un système de change à absorber les chocs nominaux (différentiel d'inflation) et les chocs réels (chocs de demande ou d'offre). Pour le second, on s'interroge sur les conséquences du choix stratégique sur la volatilité du taux de change, étant entendu qu'une variabilité excessive est jugée comme perturbatrice pour une économie très ouverte (commerce et mouvements de capitaux). On peut y ajouter la volonté de stabiliser le taux de change réel (taux nominal corrigé des écarts d'inflation entre principaux partenaires), en tout cas d'éviter une appréciation trop importante, synonyme de perte de compétitivité-prix pour les producteurs nationaux.

Les caractéristiques spécifiques des pays en développement introduisent des dimensions supplémentaires dans ce choix stratégique : vulnérabilité aux chocs externes, concentration géographique des échanges, engagements libellés en devises étrangères, taux d'inflation parfois élevé et faible crédibilité monétaire, marché domestique des capitaux souvent embryonnaire. La diversité des situations structurelles et macroéconomiques implique qu'il n'y a pas de régime de change optimal pour l'ensemble des PED. Il faut même considérer que le processus de développement nécessite une adaptation régulière du régime de change en fonction de l'étape qui est atteinte par chaque économie.

\section{Une typologie des régimes de change dans les PED}

Depuis 1998, le FMI présente une classification des régimes de change en huit catégories allant de l'absence de monnaie séparée (type dollarisation) jusqu'au flottement pur en passant par les caisses d'émission (currency board) et les différentes formes de parités fixes ou ajustables. Nous présentons ici quelques-unes de ces familles ${ }^{1}$. Dans la famille des changes fixes ou plutôt stables, il faut distinguer les cas en fonction de la monnaie et du mode de rattachement. On observe ainsi quelques situations de fixité par rapport à une seule monnaie : le dollar dans les Caraïbes, en Amérique centrale ou pour quelques îles du Pacifique, le franc français ('’euro depuis 1999) pour la Zone franc ou encore le rand sud-africain pour l'Afrique australe. Plus fréquemment, l'ancrage nominal se fait par rapport à un panier de monnaies. Les motivations principales sont commerciales, c'est-à-dire stabiliser le change par rapport aux monnaies des principaux pays partenaires, mais aussi monétaires, à savoir s'engager dans un processus crédible de désinflation. La modalité de rattachement peut admettre un ajustement régulier du taux de change nominal (crawling peg). La parité ajustable (avec ou sans bandes de fluctuation) consiste à stabiliser le taux de change réel. L'intérêt majeur est d'éviter une forte appréciation réelle dans le cas où l'ancrage s'effectue sur la monnaie d'un pays avec lequel l'écart d'inflation est initialement important. L'inconvénient est que l'engagement du gouvernement peut apparaître comme moins prononcé donc moins crédible. Pour certains le crawling peg peut devenir un "galloping peg" (Ibid., p.27), un régime de change où le rythme de dépréciation n'est plus maitrisable.

Dans la famille des changes flexibles, les systèmes de flottement impur ou administré sont les plus fréquents. Dans un tel régime, le marché des changes exerce une influence substantielle sur la détermination du taux de change. Les interventions officielles cherchent seulement à réduire les fluctuations de court terme jugées excessives ou indésirables. Le taux de change ne fournit plus d'ancrage nominal à la politique économique. Il faut donc trouver un nouvel objectif crédible à la politique monétaire (ciblage d'inflation), ce qui implique généralement des changements institutionnels (indépendance de la banque centrale). Cette stratégie ne peut convenir qu’à des pays en stade de développement avancé, ayant résolu certains problèmes structurels évoqués précédemment: finances publiques saines, systèmes financiers solides.

\footnotetext{
${ }^{(*)}$ Université d'Orléans, LEO-CNRS, thierry.montalieu @ univ-orleans.fr

${ }^{1}$ Pour les détails, voir Mussa M. et alii (2000), "Exchange rate regimes in an increasingly integrated world economy", Occasional Paper, FMI, ${ }^{\circ} 193$.
} 
Nous nous arrêtons enfin sur deux cas de figure extrêmes qui alimentent le débat depuis la fin de la décennie 1990 : le currency board et la dollarisation ${ }^{2}$. Le currency board, ou caisse d'émission, est caractérisé par trois éléments : un taux de change fixe avec la monnaie de rattachement, la convertibilité automatique et l'engagement crédible des autorités monétaires. Concrètement, il s'agit de garantir la base monétaire par les réserves de change. Les objectifs en sont la restauration de la crédibilité économique, la lutte contre l'inflation et la réduction du niveau moyen des taux d'intérêt domestiques. Par contre, ce système est peu adapté aux économies très vulnérables aux chocs extérieurs et à celles qui ont un secteur bancaire affaibli. En effet, la politique monétaire n'existe plus dans le sens où la création monétaire ne dépend que de la capacité de l'économie nationale à accroître ses réserves en devises. Dès lors, il n'est plus possible de soutenir un secteur bancaire défaillant (rôle du prêteur en dernier ressort) et d'adapter la masse monétaire aux données conjoncturelles. La politique budgétaire doit elle-même devenir prudente, puisque le financement monétaire du déficit budgétaire n'est plus envisageable. Le renoncement à la souveraineté monétaire peut aller jusqu'à l'abandon d'une monnaie nationale. Le mouvement de dollarisation, c'est-àdire l'adoption par un pays autre que les Etats-Unis du dollar comme moyen de paiement sur son territoire, n'est plus anecdotique. Quel est l'intérêt d'un tel système par rapport au schéma de caisse d'émission? Celles-ci ne suppriment pas toute attaque spéculative comme le cas argentin l'a démontré après la crise mexicaine de 1994/95 et la crise brésilienne de 1999/2000. Avec la dollarisation (ou “euroïsation") pour les pays d'Europe centrale et orientale [PECO]), l'engagement des autorités monétaires apparait plus crédible car moins facilement réversible. La disparition de la prime de risquepays associée au taux de change (le risque souverain de non-transfert demeure) doit entraîner une baisse sensible des conditions d'endettement extérieur. Par contre, une économie qui adopte une monnaie étrangère se prive de toute possibilité d'avoir une politique monétaire et de change autonome. En outre, L'État perd les recettes de seigneuriage (écart entre le pouvoir d'achat de la monnaie et son coût de production et de distribution) en renonçant au droit de battre monnaie.

\section{Une tendance à la bipolarisation}

Les nombreuses crises monétaires des années 1990 ont conduit à une remise en cause des régimes de change intermédiaires qu'il s'agisse des schémas d'ancrage souple ou des flottements fortement contrôlés. Dans le premier cas, les autorités monétaires cherchent à défendre une valeur du taux de change ou à maitriser un rythme de dépréciation préditerminé. Dans le second, la banque centrale s'efforce de préserver la stabilité du taux de change par des interventions sans s'attacher à une référence annoncée. Il semble en effet que l'ouverture croissante des économies aux flux de capitaux rende très difficile la viabilité à long terme de ce type de stratégie. Pour illustrer cette idée, l'étude de la fréquence des crises dans le cadre des différents régimes de change est riche d'enseignements ${ }^{3}$. Existe-t-il des régimes réellement prédisposés aux crises? La réponse empirique à cette question est positive. Les régimes intermédiaires sont surreprésentés dans les épisodes de crises et ce ceci est particulièrement vrai pour les économies en développement. En conséquence, de nombreux pays abandonnent progressivement ces solutions pour leur préférer des régimes extrêmes. En 1990, ces derniers ne pesaient que 30,8\% des régimes de change des pays membres du FMI ; en 2001, ce chiffre est de 61,3\%. On a donc assisté à une inversion complète de tendance. Pour autant, il convient de rappeler que les régimes extrêmes peuvent aussi être soumis à de fortes tensions (cas du rand sud-africain pour le flottement, cas du peso argentin ou $\mathrm{du}$ franc CFA pour les ancrages fixes).

\section{La transition de la fixité vers le flottement}

Nous avons observé que la période récente a été marquée par des changements souvent radicaux d'orientation des stratégies de change dans les économies émergentes. Cette transition a pu parfois s'effectuer dans la précipitation. Aussi est-il important de s'interroger sur les conditions pour une "transition ordonnée"4. Ce débat est particulièrement pertinent pour les pays qui décident d'abandonner la fixité pour aller vers le flottement de leur taux de change.

\footnotetext{
${ }^{2}$ Baliño T. et alii (1997), "Currency board arrangements : issues and experiences”, Occasional Paper, n 151 , FMI. Berg A. et Borensztein E. (2000), “Le débat sur la dollarisation ”, Finances \& Développement, mars, pp. 38-41.

${ }^{3}$ Voir l'analyse de la période 1990-2001 par Bubula A. et I. Okter-Robe (2004), « Une bipolarisation persistante », Finances \& Développement, mars, pp. 32-35.

${ }^{4}$ Pour reprendre le terme employé par Dattagupta R., G. Fernandez et C. Karacadag (2004), "From fixed to float : operational aspects of moving towards exchange rate flexibility", IMF Working Paper 04/126.
} 
Des questions opérationnelles essentielles doivent être traitées pour concevoir un passage à la flexibilité réduisant le plus possible les risques. Tout d'abord, le marché des changes doit être suffisamment profond et liquide pour une détermination efficiente des prix des monnaies; cela renvoie à des caractéristiques telles que la qualité de l'information, le niveau des restrictions réglementaires ou encore la disponibilité d'instruments de couverture. Il y a ensuite la stratégie d'intervention de la banque centrale qui, en régime de change fixe, n'a guère d'autre choix que d'intervenir fréquemment (et parfois massivement) pour soutenir la parité officielle. En régime flottant, l'intervention devient de fait discrétionnaire, ce qui laisse des marges de manœuvre pour apprécier le moment opportun et la stratégie. L'expérience semble montrer que des interventions sélectives et parcimonieuses sont préférables ; le "désalignement" du taux de change n'est pas toujours aisé à mesurer, la volatilité de court terme ne justifie pas forcément des interventions et l'efficacité de ces dernières est corrélée négativement à leur fréquence. Le cadre de la politique monétaire doit être redéfini si la stratégie de change ne fournit plus l'ancrage nominal nécessaire. La solution est, en général, d'adopter un ciblage d'inflation pour la politique monétaire. Se pose alors la question institutionnelle du statut de la banque centrale vis-à-vis du gouvernement. En terme d'agenda des réformes, un problème fondamental réside dans l'articulation entre le changement de régime de change et la libéralisation des mouvements de capitaux. Une forte libéralisation préalable a souvent été à l'origine de crises de change mais, dans le cas contraire, d'autres difficultés peuvent surgir. Le point central devient alors l'élimination des différences de traitement entre les entrées et les sorties de capitaux. En effet, cette possible asymétrie sera à l'origine d'une surévaluation (ou sous-évaluation) du taux de change. Reste le choix primordial du rythme de la transition ; une approche graduelle faite d'étapes intermédiaires (ancrage à un panier, bandes de fluctuation, ...) ou une stratégie de "big bang". La stratégie rapide a d'autant plus de chance de réussir que les institutions et les marchés sont dévéloppés et solides. D'un autre côté la démarche progressive doit s'attacher à rendre crédible la nouvelle orientation.

\section{La piste des monnaies « continentales 》}

Si les régimes extrêmes ont, semble-t-il, connu leur heure de gloire au cours des dix dernières années, il est une autre stratégie qu'il convient d'analyser : la voie des unions monétaires à une échelle régionale. La candidature de nombreux pays en transition à la zone euro ou le bilan réévalué de la Zone franc sont des illustrations de ce regain d'intérêt pour la question. De nombreuses publications récentes attestent de cette actualité5. Une monnaie supranationale accroit théoriquement la crédibilité, assure une meilleure stabilité financière et favorise l'intégration économique. Ce choix est évidemment conditionné par un certain nombre de critères concernant la structure des échanges (part du commerce intra-zone), la corrélation des conjonctures (chocs symétriques), la convergence monétaire et financière (écart de taux d'intérêt, pressions sur les marchés des changes) et des considérations d'ordre politique et institutionnel (volonté politique commune, disparités de développement) auxquels on pourrait encore ajouter les autres conditions habituelles portant sur les zones monétaires optimales. De ce point de vue, il ne paraît pas approprié aujourd'hui d'imaginer des unions monétaires à l'échelle d'un continent entier pour des économies émergentes (Amérique Latine par exemple). Cependant, l'idée d'élargir progressivement les zones monétaires existantes en sélectionnant les pays les plus appropriés, ce qui les inciterait à consolider leur politique économique (convergence nominale, ouverture commerciale,...), est une piste à creuser, en Afrique subsaharienne notamment.

\section{Une leçon de quelques expériences}

Ce numéro de Mondes en Développement propose cinq articles centrés sur les régimes de change dans les économies émergentes ou en développement. Ils offrent une diversité d'approche (théorique, empirique, historique et institutionnelle) et une large couverture géographique (Amérique Latine, Caraïbes, Afrique subsaharienne, PECO et Asie de l'Est).

La dollarisation intégrale en Amérique Latine est examinée par A. MINDA. Ce choix est souvent présenté comme la solution de la dernière chance pour les économies caractérisées par une histoire financière et monétaire mouvementée. Mais l'auteur nous montre que le terme de dollarisation recouvre une multitude de formes envisageables, ce qui le conduit à préciser une typologie des régimes de dollarisation. Les bénéfices attendus sont la crédibilité monétaire et la baisse des taux d'intérêt, la stabilité économique et la

\footnotetext{
${ }^{5}$ Voir, par exemple : Masson P. et C. Patillo (2004), The monetary geography of Africa, Brookings Institution, Washington, Berg A., E. Borensztein et P. Mauro (2003), "Quel régime monétaire pour l'Amérique Latine" Finances \& Développement, septembre, pp. 24-27.
} 
réduction de l'inflation, l'ouverture commerciale sur l'extérieur, l'approfondissement du système financier, la discipline budgétaire et enfin une forme de stabilité politique. Bien sûr ces effets bénéfiques potentiels sont à mettre en balance avec les coûts associés au processus de dollarisation. Il s'agit de la perte d'autonomie de la politique monétaire, de l'abandon de la politique de change, de la privation du droit de seigneuriage, de la disparition de la fonction de "prêteur en dernier ressort" de la banque centrale et enfin de la réduction des instruments de pilotage conjoncturel. L'article pose également la question de la responsabilité des autorités politiques et monétaires américaines dans ce processus et celle du développement des stratégies d'euroïsation dans d'autres régions du monde.

Les spécificités de la dollarisation de l'économie congolaise (RDC) sont envisagées par K. LENDELE et J. KAMANDA dans une perspective empirique. La crise politique dans ce pays a produit une désintégration graduelle de son économie dont l'une des manifestations est la perte de crédibilité de la monnaie nationale dans ses fonctions essentielles. Le caractère atypique de la dollarisation au Congo est d'abord souligné en montrant qu'il s'agit surtout d'une substitution pure de monnaies, alors que la substitution d'actifs financiers reste marginale. Les résidents ont donc pris l'habitude de facturer les produits et les services en devises (surtout dollar), de même pour la rédaction des contrats commerciaux. L'État lui-même a été amené à accepter le paiement des taxes en devises. Une démarche économétrique pour estimer le degré de dollarisation de l'économie congolaise et en asseoir les déterminants est ensuite entreprise. Le taux de dollarisation (calculé officiellement) de l'économie se serait fortement accru de 7\% en 1990 à 45\% en 2003. Mais la réalité mesurée par les auteurs est plus intense : leur propre ratio passe de $15 \%$ à $84 \%$ sur la même période. Les anticipations inflationnistes des agents sont bien l'argument essentiel du développement de cette dollarisation. Les effets négatifs sur le seigneuriage sont également mis en évidence.

Dans une démarche qui allie analyse théorique et tests économétriques, R. VEYRUNE s'intéresse au "prix de la fixité » dans le cas des économies ayant conclu des accords de type «union de taux de change ». Les coûts de l'abandon de la flexibilité du change sont appliqués au cas des pays de l'Union monétaire des Caraỉbes orientales et à la Zone franc. A l'aide d'un outillage analytique qui emprunte beaucoup à la modélisation des petites économies ouvertes (Salter-Swan, Edwards), l'auteur détermine les enjeux de politique économique liés à l'abandon de la flexibilité des changes. Il s'agit, pour l'essentiel, d'apprécier l'ampleur des coûts de l'ajustement passif du change réel : perte de seigneuriage, récession, désintermédiation et effets redistributifs. La question est aussi de déterminer les facteurs qui influencent l'ampleur de ces coûts (flexibilité des prix, capacité d'emprunt en devises). Sur le plan économétrique, une analyse en données de panel est menée. Les écarts conjoncturels de la croissance du produit réel sont régressés sur la déviation du taux de change (désalignement par rapport à l'équilibre) et des variables de contrôle (ouverture, capacité d'endettement, réserves en devises).

Dans une perspective qui emprunte beaucoup à l'analyse historique, J.B. DESQUILBET et N. NENOVSKY présentent une comparaison des régimes d'étalon-or et de caisse d'émission. En effet, ces deux stratégies de change ont pu être assimilées par certains, puisqu'elles impliquent des règles monétaires très contraignantes. Cette analyse comparative est menée d'un double point de vue : les sources de la confiance et les mécanismes d'ajustement. Dans un premier temps, l'efficacité d'un régime monétaire est évaluée à l'aune de sa capacité à promouvoir la confiance à travers la convertibilité totale, son processus de constitution (endogène pour l'étalon-or ou exogène pour la caisse d'émission) et le contexte international dans lequel le régime évolue (arrangement international, pression des institutions financières internationales). Il s'agit, par la suite, d'identifier la capacité des deux régimes à résister aux chocs par les mécanismes d'ajustement qu'ils suscitent. Le caractère automatique de l'ajustement, l'évolution de l'environnement financier (innovations monétaires, globalisation financière, croissance de l'intermédiation bancaire,...) et la présence d'asymétries de réaction des acteurs (pays dominants et dominés) en sont les principaux déterminants. Les auteurs montrent, notamment, que les deux régimes peuvent contenir des éléments discrétionnaires et le régime de caisse d'émission correspond plutôt à la situation des pays périphériques de l'étalon-or. De ce point de vue, ils sont amenés à supporter la totalité du poids de l'ajustement.

K.S. LEE et P. SAUCIER, pour leur part, cherchent à faire le lien entre stratégie monétaire et enjeux commerciaux dans le contexte des économies de l'est asiatique. Au regard de la théorie des zones monétaires optimales, la région ne semble pas être en mesure d'absorber les chocs sans ajustement des taux de change réciproques. Pour autant, faut-il renoncer à toute tentative de stabilisation des taux de 
change bilatéraux ? Sur un plan empirique, l'influence de la volatilité des taux de change est évaluée à l'aide d'un modèle explicatif du commerce bilatéral appliqué à huit économies de la zone. La méthodologie s'inspire des approches gravitationnelles et nécessite, pour ce qui est des taux de change, de mesurer leur volatilité (modélisation ARCH). Ce travail apporte une contribution supplémentaire au débat récurrent sur la coopération monétaire en zone Asie, dans le sens où il confirme que la réduction de l'instabilité des changes serait favorable à la densification des échanges intra-régionaux. Dans cet esprit, la stratégie minimaliste poursuivie jusqu'à présent en matière d'intégration régionale dans cette zone peut désormais être considérée comme insuffisante pour franchir un palier supplémentaire.

Les différentes expériences relatées dans ce numéro soulignent encore une fois qu'il n'y a pas de stratégie de change ou de régime monétaire préférable dans l'absolu. Par contre, les caractéristiques d'une économie, les priorités données à la politique économique éclairent ce choix ; ces déterminants peuvent avoir un caractère évolutif. Toute la difficulté consiste alors à organiser la phase de transition d'un régime à l'autre (sortie de caisse d'émission, fixité vers flexibilité...), afin de réduire autant que possible les coûts associés à cette transition. 